\title{
A tiling approach to Fibonacci product identities
}

\author{
Jacob Artz and Michael Rowell \\ (Communicated by Arthur T. Benjamin)
}

In 1998 Filipponi and Hart introduced a number of Fibonacci product identities. This paper provides a combinatorial proof for such identities via tilings. The methods used in the proof are then further used to produce some new Zeckendorf representations and a known Fibonacci identity.

\section{Introduction}

The discovery of the Fibonacci sequence is credited to Leonardo of Pisa (c. 11701250), posthumously nicknamed Fibonacci ("son of Bonaccio"). He is said to have come upon it while considering the breeding of rabbits [Russel 2008]. We define the Fibonacci sequence recursively.

Definition 1.1. Let $f_{0}=1, f_{1}=1$. For $n \geq 2$, set $f_{n}=f_{n-1}+f_{n-2}$. We say that $f_{n}$ is the $n$-th Fibonacci number.

The first few terms of the Fibonacci sequence are 1, 1, 2, 3, 5, 8, 13, 21,... Fibonacci numbers can also be explicitly defined, although the statement of each term is somewhat less elegant than its recursive counterpart:

$$
F_{n}=\frac{1}{\sqrt{5}}\left[\left(\frac{1+\sqrt{5}}{2}\right)^{n}-\left(\frac{1-\sqrt{5}}{2}\right)^{n}\right] .
$$

For the purpose of this paper, we introduce an interpretation of the Fibonacci sequence which allows us to combinatorially prove Fibonacci identities. We will interpret the $n$-th Fibonacci number as the number of tilings of a $1 \times n$ board using $1 \times$ 1 squares and $1 \times 2$ dominoes. Thus, $\left(f_{0}, f_{1}, f_{2}, f_{3}, f_{4}, \ldots\right)=(1,1,2,3,5, \ldots)$. (For more on this interpretation, see [Benjamin and Quinn 2003].)

Zeckendorf's Theorem says that any positive integer $n$ can be represented as a sum of distinct, nonconsecutive Fibonacci numbers, excluding $f_{0}$. This was first published in [Lekkerkerker 1952], though it had been proved by Zeckendorf many years before. Finding the Zeckendorf representation of a particular number $n$ is in

MSC2000: 05A19, 11B39.

Keywords: Fibonacci products, tiling. 
fact easy: starting from $n$, successively subtract the largest Fibonacci number that will fit. (With some thinking this also justifies the theorem: basically, repeats are impossible because $f_{n} \leq 2 f_{n-1}$, so what's less at each stage is strictly less than what was just subtracted; and consecutive Fibonacci numbers don't occur, because if $f_{n}$ and $f_{n+1}$ occurred one would instead have used $f_{n+2}$. See also [Brown 1964].)

It is an interesting problem to find explicitly the Zeckendorf representation of various numbers. For example, the following identities are found in [Filipponi and Hart 1998]:

Theorem 1.2. For $k \geq 0$ and $n \geq 2 k$,

$$
f_{2 k+1} f_{n}=f_{n+2 k}+f_{n+2 k-4}+\cdots+f_{n-2 k+4}+f_{n-2 k}=\sum_{i=0}^{k} f_{n+2 k-4 i} .
$$

Theorem 1.3. For $k \geq 1$ and $n \geq 2 k$,

$$
f_{2 k} f_{n}=f_{n+2 k-1}+f_{n+2 k-5}+\cdots+f_{n-2 k+3}+f_{n-2 k}=\sum_{i=0}^{k-1} f_{n+2 k-1-4 i}+f_{n-2 k} .
$$

Wood [2007] presents combinatorial proofs for the expansion of $f_{4} f_{n}$ and $f_{5} f_{n}$, but no unifying counting argument is shown. Gerdemann [2009] provides a combinatorial proof of the existence of Zeckendorf representations but, due to the algorithmic nature of the proof, is unable to produce closed form identities like the Fibonacci products provided above.

In the sequel we give examples of how tilings of one-row boards can help prove Fibonacci identities, starting with simple examples in Section 2 and continuing with provide combinatorial proofs for Theorems 1.2 and 1.3 in Section 3. In Section 4, we use the methods from Section 3 to find new Zeckendorf representations and a known Fibonacci identity. Directions for future research are given in Section 5.

\section{Basic methods of tiling}

A cell is a space of length one appearing on a $1 \times n$ board. While the meaning of this term may seem obvious, we wish to eradicate any confusion with the term tile, defined as a one- or two-cell piece used to create a tiling of the board.

We now illustrate two basic methods of showing Fibonacci identities using tilings; see Benjamin and Quinn [2003].

Considering the location of a fault. A fault in a tiling is the coordinate of any boundary between tiles. For example, in this tiling of length 5 with 3 tiles, the faults are at positions 1 and 3:

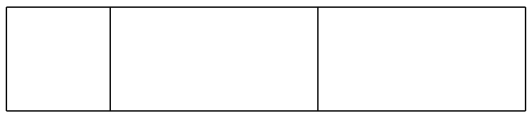


Theorem 2.1. For $n, m \geq 1$,

$$
f_{m+n}=f_{m} f_{n}+f_{m-1} f_{n-1} .
$$

Proof. The left side of our equation, $f_{m+n}$, is the number of ways of tiling a board of length $m+n$.

To interpret the right side, we consider the cell at position $m$ of a board of length $m+n$. If the board has a fault at $m$, then there are $f_{m}$ ways to tile the first $m$ tiles, and $f_{n}$ ways to tile the remaining $n$ tiles. Therefore, there are $f_{m} f_{n}$ ways to tile the entire board. If the board does not have a fault at $m$, then there must be a domino covering cells $m$ and $m+1$. Using similar reasoning as in our previous case, there are $f_{m-1} f_{n-1}$ ways to tile the entire board. Therefore, there are a total of $f_{m} f_{n}+f_{m-1} f_{n-1}$ ways to tile a board of length $m+n$.

Finding correspondences. In the second method, we show identities by interpreting a side as multiple copies of board. We again give illustrate the method by giving the proof of another basic identity.

Theorem 2.2. For $n \geq 2$,

$$
2 f_{n}=f_{n+1}+f_{n-2} .
$$

Proof. We will show that two copies of each tiling of length $n$ can be mapped to the set counted by tilings of length $n+1$ and those of length $n-2$. Begin by examining our first copies of tilings of length $n$. We append a square to each of our length- $n$ tilings to form the set of all boards length of $n+1$ that end in a square tile. We now consider our second copies of our length- $n$ tiling. If the last tile is a square, remove the last square and add a domino, resulting in all $n+1$ tilings that end in a domino. If the last tile is a domino, then remove the domino to form all tilings of length $n-2$.

\section{Fibonacci products and tiling}

Before tackling Theorems 1.2 and 1.3, we begin with proving a simple, yet very useful identity,

Theorem 3.1. For $k, n \geq 2$,

$$
f_{k} f_{n}=f_{n+k-1}+f_{k-2} f_{n-2} .
$$

Proof. We begin by noting that the left side counts the number of tilings of length $n+k$ that have a fault at $n$.

We then note that $f_{n-2} f_{k-2}$ counts the number of tilings of length $n+k-4$ that have a fault at $n-2$. Inserting two dominoes between cells $n-2$ and $n-1$ yields all tilings of length $n+k$ with a fault at $n$ and with dominoes on both sides of the fault. 
We now inspect $f_{n+k-1}$, all tilings of a board length $n+k-1$. For all tilings that do not have a fault at $n$ (implying there is a fault at $n-1$ ), we insert a square between cells $n-1$ and $n$, creating all tilings of length $n+k$ with a fault at $n$ and with a square to the left of the fault and a domino to the right. For all tilings that $d o$ have a fault at $n$, we insert a square between cells $n$ and $n+1$, creating all tilings of length $n+k$ with a fault at $n$ and a square to the right of the fault.

The set of all tilings of length $n+k$ with a fault at $n$ is exactly equal to the three cases defined above.

We can now see that inductively applying this theorem to the term $f_{k} f_{n}$ will allow us to combinatorially prove Theorems 1.2 and 1.3. For example $(n \geq 6)$,

$$
\begin{aligned}
f_{7} f_{n} & =f_{n+6}+f_{5} f_{n-2}=f_{n+6}+f_{n+2}+f_{3} f_{n-4} \\
& =f_{n+6}+f_{n+2}+f_{n-2}+f_{1} f_{n-6}=f_{n+6}+f_{n+2}+f_{n-2}+f_{n-6} .
\end{aligned}
$$

Proof of Theorem 1.2. The theorem is trivial for $k=0$. Assuming the theorem is true for $k \leq m$, we see that

$$
\begin{aligned}
f_{2(m+1)+1} f_{n} & =f_{n+2 m+2}+f_{2 m+1} f_{n-2} \quad \text { by Theorem } 3.1 \\
& =f_{n+2 m+2}+\sum_{i=0}^{m} f_{n+2 m-2-4 i} \text { by the inductive hypothesis } \\
& =\sum_{i=0}^{m+1} f_{n+2 m+2-4 i} .
\end{aligned}
$$

Proof of Theorem 1.3. In the case $k=1$, our theorem reduces to Theorem 2.2. Assuming that the theorem is true for $k \leq m$, we see that

$$
\begin{aligned}
f_{2(m+1)} f_{n} & =f_{n+2 m+1}+f_{2 m} f_{n-2} \quad \text { by Theorem } 3.1 \\
& =f_{n+2 m+1}+\sum_{i=0}^{m-1} f_{n+2 m+1-4 i}+f_{n-2 m} \text { by inductive hypothesis } \\
& =\sum_{i=0}^{m} f_{n+2 m+1-4 i}+f_{n-2 m} .
\end{aligned}
$$

We now have a unifying combinatorial proof for the following identities:

$$
\begin{aligned}
& f_{2} f_{n}=f_{n+1}+f_{n-2}, \\
& f_{3} f_{n}=f_{n+2}+f_{n-2}, \\
& f_{4} f_{n}=f_{n+3}+f_{n-1}+f_{n-4}, \\
& f_{5} f_{n}=f_{n+4}+f_{n}+f_{n-4}, \\
& f_{6} f_{n}=f_{n+5}+f_{n+1}+f_{n-3}+f_{n-6}, \\
& f_{7} f_{n}=f_{n+6}+f_{n+2}+f_{n-2}+f_{n-6},
\end{aligned}
$$


We note that the proofs of Theorems 1.2 and 1.3 need not rely on induction. In Theorem 1.2, the term $f_{n+2 k-4 i}$ can be interpreted as all tilings of length $n+2 k+1$ that have a fault at $n$ and whose nearest square tile to the fault is exactly $2 i$ cells away. Because $2 k+1$ is odd, we are guaranteed to have a square tile within at most $2 k$ of the fault, thus $i$ ranges from 0 to $k$. Theorem 1.3 is slightly different in that we are not guaranteed to have a square tile within $2 k-2$ of our fault. Thus, we must add the term $f_{n-2 k}$ to account for all tilings of length $n+2 k$ with a square no closer than $2 k$ cells from the fault at $n$.

\section{Further observations}

Using Theorem 3.1, we can determine other closed form Zeckendorf representations and a known Fibonacci identity.

Lemma 4.1. For $k \geq 1$ and $n \geq 2 k$,

$$
\left(f_{2 k}+f_{2 k-2}\right) f_{n}=f_{n+2 k}+f_{n-2 k} .
$$

Proof. In the case $k=1$, our lemma reduces to the case $k=1$ in Theorem 1.2. Assuming our lemma is true for $k \leq m$ and applying Theorem 3.1 we see that

$$
\begin{aligned}
\left(f_{2 m+2}+f_{2 m}\right) f_{n} & =f_{n+2 m+1}+f_{n+2 m-1}+\left(f_{2 m}+f_{2 m-2}\right) f_{n-2} \\
& =f_{n+2 m+1}+f_{n+2 m-1}+f_{n+2 m-2}+f_{n-2 m-2} \\
& =f_{n+2 m+2}+f_{n-2 m-2},
\end{aligned}
$$

where our second to last line follows from the inductive hypothesis, and the last line follows from the recursive definition of the Fibonacci sequence.

As with our two main theorems, we can prove Lemma 4.1 without induction. Using Theorem 1.3 we see that

$$
\left(f_{2 k}+f_{2 k-2}\right) f_{n}=\sum_{i=0}^{2 k-2} f_{2 k-1+n-2 i}+f_{n-2 k+2}+f_{n-2 k} .
$$

It is left to show that

$$
f_{n+2 k}=\sum_{i=0}^{2 k-2} f_{2 k-1+n-2 i}+f_{n-2 k+2} .
$$

This can be done by considering the position of the last square. Note that $f_{2 k-1+n-2 i}$ counts the number of tilings of length $n+2 k$ with the last square in the $(n+2 k+$ $1-2 i$ ) cell followed by dominoes. Our sum accounts for all tilings of length $n+2 k$ with the last square appearing somewhere past the $(n-2 k+2)$ cell. We then see that $f_{n-2 k+2}$ counts the remaining tilings of length $n+2 k$, namely those which end in $k-1$ dominoes. 
Lemma 4.2. For $k \geq 1$ and $n>2 k$,

$$
\left(f_{2 k-1}+f_{2 k+1}\right) f_{n}=f_{n+2 k+1}-f_{n-2 k-1} .
$$

Proof. In the case $k=1$, we repeatedly use our recursive definition of the Fibonacci sequence,

$$
\begin{aligned}
f_{n+3}-4 f_{n} & =\left(f_{n+2}+f_{n+1}\right)-4 f_{n}=\left(f_{n+1}+f_{n}\right)+\left(f_{n}+f_{n-1}\right)-4 f_{n} \\
& =\left(\left(f_{n}+f_{n-1}\right)+f_{n}\right)+\left(f_{n}+f_{n-1}\right)-4 f_{n}=-f_{n}+2 f_{n-1}=f_{n-3},
\end{aligned}
$$

where our last line follows from Theorem 2.2. Assuming our lemma is true for $k \leq m$ and applying Theorem 3.1 we see that

$$
\begin{aligned}
\left(f_{2 m+1}+f_{2 m+3}\right) f_{n} & =f_{2 m+n}+f_{2 m-1} f_{n-2}+f_{2 m+2+n}+f_{2 m+1} f_{n-2} \\
& =f_{n+2 m}+f_{n+2 m+2}+f_{n+2 m-1}-f_{n-2 m-3} \\
& =f_{n+2 m+3}-f_{n-2 m-3},
\end{aligned}
$$

where our second to last line follows from using our inductive hypothesis and our last line follows from the recursive definition of the Fibonacci sequence.

Using Theorem 1.2 and Lemma 4.1 we can now give the Zeckendorf representation of the following family of identities:

$$
\begin{aligned}
3 f_{n} & =\left(f_{0}+f_{2}\right) f_{n}=f_{n+2}+f_{n-2}, \\
4 f_{n} & =\left(f_{1}+f_{3}\right) f_{n}=f_{n+2}+f_{n}+f_{n-2}, \\
7 f_{n} & =\left(f_{2}+f_{4}\right) f_{n}=f_{n+4}+f_{n-4}, \\
11 f_{n} & =\left(f_{3}+f_{5}\right) f_{n}=f_{n+4}+f_{n+2}+f_{n}+f_{n-2}+f_{n-4}, \\
18 f_{n} & =\left(f_{4}+f_{6}\right) f_{n}=f_{n+6}+f_{n-6},
\end{aligned}
$$

Combining Lemmas 4.1 and 4.2 we obtain our Fibonacci identity, which appears in a more general form as Identity 48 in [Benjamin and Quinn 2003].

Theorem 4.3. For $k \geq 1$ and $n>k$,

$$
\left(f_{k+1}+f_{k-1}\right) f_{n}=f_{n+k+1}-(-1)^{k} f_{n-k-1} .
$$

\section{Future work}

As previously mentioned, Theorems 1.2 and 1.3 first appeared in [Filipponi and Hart 1998] with some other Fibonacci products. The authors also presented Zeckendorf representations for $2 f_{k} f_{n}, 3 f_{k} f_{n}, 4 f_{k} f_{n}$ and $5 f_{k} f_{n}$. It is not difficult to see that these formulas can also be obtained using our counting method presented above. One only needs to determine the appropriate recurrence relation. 
Also, while the proofs in Section 4 relied on the use of Theorem 3.1, it is not entirely clear how to construct a counting proof for Theorem 4.3. It would be interesting to see a combinatorial proof of the identity.

\section{Acknowledgment}

The authors would like to thank the reviewers for their comments and suggestions. In particular, it was quite helpful to consider combinatorial proofs without the use of induction.

\section{References}

[Benjamin and Quinn 2003] A. T. Benjamin and J. J. Quinn, Proofs that really count: The art of combinatorial proof, The Dolciani Mathematical Expositions 27, Mathematical Association of America, Washington, DC, 2003. MR 2004f:05001 Zbl 1044.11001

[Brown 1964] J. L. Brown, "A new characterization of the Fibonacci numbers", Fibonacci Quart. 2 (1964), 163-168.

[Filipponi and Hart 1998] P. Filipponi and E. L. Hart, "The Zeckendorf decomposition of certain Fibonacci-Lucas products”, Fibonacci Quart. 36:3 (1998), 240-247. MR 99d:11006 Zbl 0942. 11012

[Gerdemann 2009] D. Gerdemann, "Combinatorial proofs of Zeckendorf family identities", Fibonacci Quart. 46/47:3 (2009), 249-261.

[Lekkerkerker 1952] C. G. Lekkerkerker, "Voorstelling van natuurlijke getallen door een som van getallen van Fibonacci”, Simon Stevin 29 (1952), 190-195. MR 15,401c

[Russel 2008] D. Russel, "A short biography of Leonardo Pisano Fibonacci”, 2008, Available at http://math.about.com/od/mathematicians/a/fibonacci.htm.

[Wood 2007] P. M. Wood, "Bijective proofs for Fibonacci identities related to Zeckendorf's theorem”, Fibonacci Quart. 45:2 (2007), 138-145 (2008). MR 2009b:05032 Zbl 1162.11014

Received: 2009-08-04 Revised: 2009-09-14 Accepted: 2009-09-19

artz8028@pacificu.edu Mathematics Department, Pacific University, 2043 College Way, Forest Grove, OR 97116, United States

rowell@pacificu.edu

Mathematics Department, Pacific University, 2043 College Way, Forest Grove, OR 97116, United States www. math. pacificu.edu/ rowell 